\title{
Automatic Segmentation of Lung Fields in Chest Radiographs
}

\author{
Bram van Ginneken and Bart M. ter Haar Romeny \\ Image Sciences Institute, Utrecht University, The Netherlands \\ bram@isi.uu.nl \\ http://www.isi.uu.nl/
}

\begin{abstract}
We present algorithms for the automatic delineation of lung fields in chest radiographs. We first develop a rule-based scheme that detects lung contours using a general framework for the detection of oriented edges and ridges. This algorithm is compared to several pixel classifiers using different combinations of features. We propose a hybrid system that combines both approaches. The performance of each system is compared with interobserver variability and results available from the literature. Our hybrid scheme turns out to be accurate and robust; the accuracy is $0.969 \pm 0.00803$, and above $94 \%$ for all 115 test images.
\end{abstract}

\section{Introduction}

Chest radiographs make up 40 percent of all conventional diagnostic radiographic procedures in general hospitals [1]. The advent of digital thorax units makes it in principle possible to use computerized methods for the analysis of chest radiographs on a routine basis. Our particular interest is the development of tools for computer assistance in mass chest screening against tuberculosis.

We focus on segmentation of lung fields in standard PA chest radiographs. Automatic segmentation is a mandatory pre-processing step for computer analysis of thorax images and has received considerable attention in recent literature. The two main approaches are rule-based reasoning and pixel classification with neural networks. Rule-based schemes have been proposed by $\mathrm{Xu}$ et al. [2,3], Duryea and Boone [4], and Carrascal et al. [5]. Lung segmentation by pixel classification has been investigated by McNitt-Gray [6,7], Hasegawa et al. [8], Tsujii et al. [9], and Vittitoe et al. [10].

We consider both a rule-based system and pixel classification with several sets of features. The rule-based scheme we present is new and employs a general and flexible framework for detection of subdimensional structures. Furthermore, we propose a new hybrid segmentation scheme that combines the rule-based approach with pixel classification. We show that this system compares favourably with literature results and approaches the accuracy of segmentation by hand. Such direct comparisons between segmentation methods have not been made before. 


\section{Materials and Methods}

Our database consists of standard PA chest radiographs from a tuberculosis screening program for people seeking political asylum in The Netherlands. We randomly selected normal and abnormal cases of adult subjects. Images were taken with a mobile Electrodelca (Oldelft BV, Delft, The Netherlands), a system commonly used in mass chest screening. Tube voltage was $117 \mathrm{kV}$ and the images were printed on 10 by $10 \mathrm{~cm}$. film. The films were digitized with a Lumisys 100 scanner (Lumisys, Inc., Sunnyvale, CA) to 996 by 996 pixels with 10 bit intensity, and subsampled to 256 by 256 pixels, which is sufficient for our purposes. Images were randomly divided in a test set and a training set, each containing 115 images. We indicate the coordinate of the upper left corner of the image with $(0,0)$ and the lower right with $(1,1)$ and express scales accordingly.

All software for displaying and drawing the digitized radiographs and the segmentation algorithms presented here was written by the first author in $\mathrm{C}++$ using Borland $\mathrm{C}++$ Builder (Borland International Inc.) and runs on a $\mathrm{PC}$ with Windows NT 4.0. The lung fields were traced with a mouse by each author independently, under supervision of an experienced radiologist.

\section{Lung Segmentation Algorithms}

\subsection{Rule-Based Detection of Lung Contours}

Starting point of our rule-based approach is the observation that the borders between anatomical structures in chest radiographs largely coincide with edges and ridges in the image. A difficulty is that these structures are highly connected and correspond only partly to the borders between anatomical regions. It is not straightforward to pick out the "correct" structures or parts of structures. We propose to use directional derivatives to overcome this problem.

Edges are usually defined as those points in an image where the gradient magnitude is maximum in the gradient direction. Ridges are commonly defined as extrema in the direction of the largest curvature. Instead of these definitions, we consider structures defined by extrema in a fixed direction $\alpha$, for derivatives of a certain order in that same direction. In this way, we obtain structures that cannot cross, because in the direction $\alpha$ consecutive points cannot both be extremal. The order of derivative $n$ and the choice for minima and maxima determines the nature of the detected structures. Using order $n=0$, one detects axes of bright and dark blobs; $n=1$ yields edges from dark to bright regions, or vice versa; $n=2$ gives bright and dark line structures, all in the direction perpendicular to $\alpha$. The problem of connected structures is much reduced in this way. To solve the problem of selecting the proper structures, we use a straightforward voting technique. For the detection of diaphragm and lung top, voting mechanisms may fail to detect the correct structure. Since the lung top and diaphragm are generally the strongest lines and edges, we used dynamic programming to detect them. Putting this all together, estimates of the diaphragm, the border between lung fields and mediastinum, the boundaries of the rib cage and the lung tops 


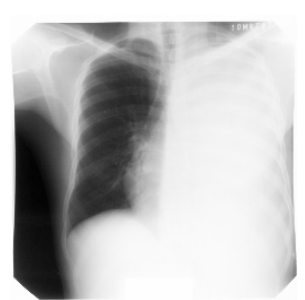

(a)

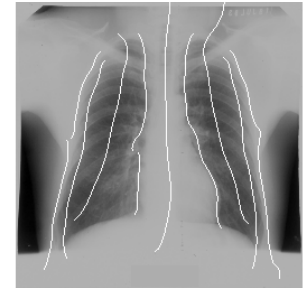

(b)

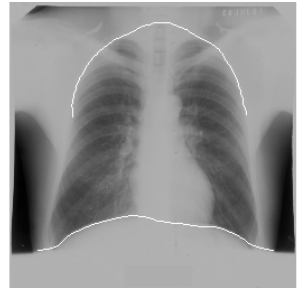

(c)

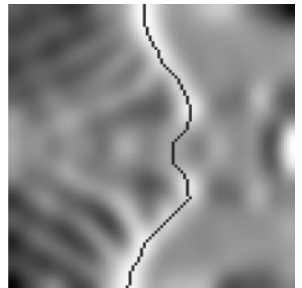

(d)

Fig. 1. (a) Example of an image that is rejected because no thorax center line is found; (b) Typical result of detection of vertical structures (thorax center line, mediastinum edges, lung edges, rib cage); (c) Typical result of detected diaphragm and lung top detected; (d) Polar transformation of a hemicircle of the image used in the detection of the lung top. The horizontal axis is the radius, the vertical is the polar angle; the center of the circle is at half height on the left of the image.

are obtained. Figure 1 shows representative results. We now give a detailed description of the algorithm.

\section{System 1. Rule-based reasoning}

1. Detection of thorax center line. ROI is a rectangle defined by $0.3<x<0.7$ and $0.2<y<0.6$. This $y$-range is also used in steps $2-5$. The 0 th order derivative is computed at scale 0.05. Pixels which are maximal in the horizontal direction are detected and grouped into 8-connected structures. For each horizontal line in the ROI, the structure closest to the vertical centerline of the ROI is voted for. The structure that receives most votes is selected. Other structures are added, in order of received votes, as long as they don't overlap in their $y$-coordinates. If no center line is detected, the image is rejected.

2. Detection of the right/left edge of mediastinum. ROI is bounded on the right/left by the thorax center line and has 0.4 width. The 1 st derivative in the $x$-direction is computed at a scale of 0.03 . Maxima/minima in the horizontal direction are grouped into connected structures. For each horizontal line, starting from the center line, the first structure encountered is voted for. Again, other structures are added, in order of received votes, as long as they don't overlap in their $y$-coordinates. The same voting procedure is used in steps 3 to 5 .

3. Detection of lung center lines. The mediastinum edges are the right and left boundaries of the ROI, which has a 0.3 width. We look for horizontal minima of the 0 th order derivative at a scale of 0.03 .

4. Detection of right/left lung edges. The lung center lines are the right and left boundaries of the ROI which has a 0.4 width. We look for horizontal minima/maxima of the 1 st order derivative in the $x$-direction at a scale of 0.03 .

5. Detection of right/left rib cage. The lung edges are the right/left boundaries of the ROI which has a 0.2 width. We look for horizontal maxima of the 2nd order derivative at a scale of 0.02 . 
6. Detection of diaphragm. The right and left diaphragm are determined with dynamic programming to find a line from left to right in a rectangular ROI. The ROI contains the 1st derivative in the $y$-direction of the image computed at a scale of 0.02 . The line is allowed to have a slope between -1 and 1 . The right diaphragm is detected first, because it is visible more clearly, owing to the absence of heart and stomach bubbles. The $x$-range of the ROI is from the lowest $x$-coordinate of the rib cage to the lowest $x$-coordinate of the thorax center line. We find a point on the right diaphragm by taking the right lung center at $y=0.40$, moving down from this point to a maximum in the 1 st derivative. The $y$-range of the diaphragm is 0.4 , centred around this point. After the right diaphragm detection, crossings with the right rib cage and mediastinum edge are determined. These landmark points are added to the right diaphragm starting point. The $y$-range of the ROI for the left diaphragm is given by the smallest $y$-coordinate of these points minus 0.2 and the lowest $y$-coordinate plus 0.2 . The $x$-range is from the highest $x$-coordinate of the thorax center line to the highest $x$-coordinate of the left rib cage.

7. Detection of lung tops. We use the fact that the top is a bright line structure, just like the rib cage. It is more or less circular, so we make a polar transformation of a hemicircle in the image. As center point we take the thorax center line at $y=0.40$. The radius of the hemicircle is estimated to be half the distance between the right and left rib cage at this height. Minimum and maximum radius are set at 0.7 and 1.3 times this radius. We take the 2 nd derivative along the circle radius and find a maximum path with dynamic programming. In this path, we allow deviations from a circular path of at most $45^{\circ}$.

8. Lung contours from a reference image with 15 control points along each contour are moved in horizontal or vertical direction until they coincide with the detected mediastinum, lung top, rib cage or diaphragm respectively. The contour interpolated based on the displacements of control points is taken as final lung contour.

\section{System 2-7. Pixel classifiers}

Segmentation can also be treated as a pixel classification problem by calculating a feature vector for each pixel in the input image. Output is the anatomical class the pixel belongs to. Although different types of classifiers will obviously lead to different results, the performance of these segmentation algorithms will depend mostly on the features of the input vector. As features we use pixel location, pixel intensity, entropy, and the corrected location computed from a scaling and translation computed from the rule based scheme. The entropy measure is motivated by the fact that the lung fields contain overlying ribs and lung vasculature and are therefore less homogeneous than areas outside the lungs. It is computed by subtracting a slightly blurred version of the image from the original, taking the absolute value of this image and blurring this slightly.

We use $k$-nearest-neighbour classification with $k=31$. This setting of $k$ was determined as optimal in several pilot experiments. We used 1024 points from each image in the training set (32 by 32 ). In all systems, scaling factors were 


\begin{tabular}{|l|lll|}
\hline Method & Accuracy & Sensitivity & Specificity \\
\hline Interobserver variability & $0.984 \pm 0.00475$ & $0.957 \pm 0.0174$ & $0.993 \pm 0.00306$ \\
Classification correction (8) & $0.969 \pm 0.00803$ & $0.943 \pm 0.0330$ & $0.978 \pm 0.0106$ \\
Rule-based (1) & $0.961 \pm 0.0116$ & $0.940 \pm 0.0389$ & $0.969 \pm 0.0153$ \\
PC int., entropy, corr. location (7) & $0.956 \pm 0.0157$ & $0.912 \pm 0.0617$ & $0.972 \pm 0.0248$ \\
PC int., corrected location (6) & $0.953 \pm 0.0177$ & $0.906 \pm 0.0649$ & $0.970 \pm 0.0288$ \\
PC int. and location (5) & $0.933 \pm 0.0219$ & $0.854 \pm 0.0771$ & $0.966 \pm 0.0309$ \\
PC location (3) & $0.898 \pm 0.0382$ & $0.784 \pm 0.0984$ & $0.947 \pm 0.0339$ \\
PC int. (4) & $0.847 \pm 0.0356$ & $0.727 \pm 0.122$ & $0.891 \pm 0.0447$ \\
All negative (2) & $0.736 \pm 0.0551$ & $0 \pm 0$ & $1 \pm 0$ \\
\hline Duryea's rule-based method [4] & $0.959 \pm 0.054$ & $0.863 \pm 0.11$ & $0.987 \pm 0.044$ \\
Vittitoe's MRF [10] & $0.948 \pm 0.016$ & $0.907 \pm 0.044$ & $0.972 \pm 0.020$ \\
McNitt-Gray's 59 features [7] & 0.932 & 0.949 & 0.922 \\
Tsujii's PC corr. int., location [9] & 0.923 & & \\
McNitt-Gray's 8 features [7] & 0.918 & 0.903 & 0.930 \\
Vittitoe's PC int. 3x3 [10] & $0.893 \pm 0.027$ & $0.846 \pm 0.057$ & $0.925 \pm 0.038$ \\
Vittitoe's PC location [10] & $0.880 \pm 0.035$ & $0.820 \pm 0.08$ & $0.920 \pm 0.044$ \\
Duryea's classifying location [4] & $0.879 \pm 0.063$ & $0.785 \pm 0.10$ & $0.934 \pm 0.063$ \\
Vittitoe's fixed thresholding [10] & $0.806 \pm 0.071$ & $0.860 \pm 0.058$ & $0.781 \pm 0.112$ \\
Duryea's all negative [4] & $0.751 \pm 0.063$ & $0 \pm 0$ & $1 \pm 0$ \\
\hline
\end{tabular}

Table 1. Accuracy, sensitivity and specificity of all 8 systems on the complete test set. PC stands for pixel classification. The number in parentheses denotes the system number. The systems are sorted according to accuracy. Below the line are results from literature for which the same performance measures could be found or calculated. Note that the test sets in these studies are different.

calculated to obtain zero mean and unit standard deviation for each feature over the whole training set. These scaling factors were also applied to the features of pixels in the test images.

System 2 uses no features and thus classifies each pixel using the most likely class, i.e. the background. System 3 uses pixel location, System 4 uses intensity (a multiple threshold), System 5 uses location and intensity, System 6 uses intensity and corrected location, System 7 uses all features.

\section{System 8. Rule-based reasoning and correction using pixel classification}

This system reclassifies those pixels in the image for which systems 1 and 6 yield different classes. There are 2 possibilities, pixels can be classified as lung by system 1 and as non-lung by system 6 , or vice versa. For each case, we determine a training set consisting of all the pixels in the training set for which this situation occurs. We use corrected location as feature.

\section{Results and Discussion}

Table 1 lists the results of our schemes together with results reported in the literature, in terms of accuracy, sensitivity and specificity. We found no significant 


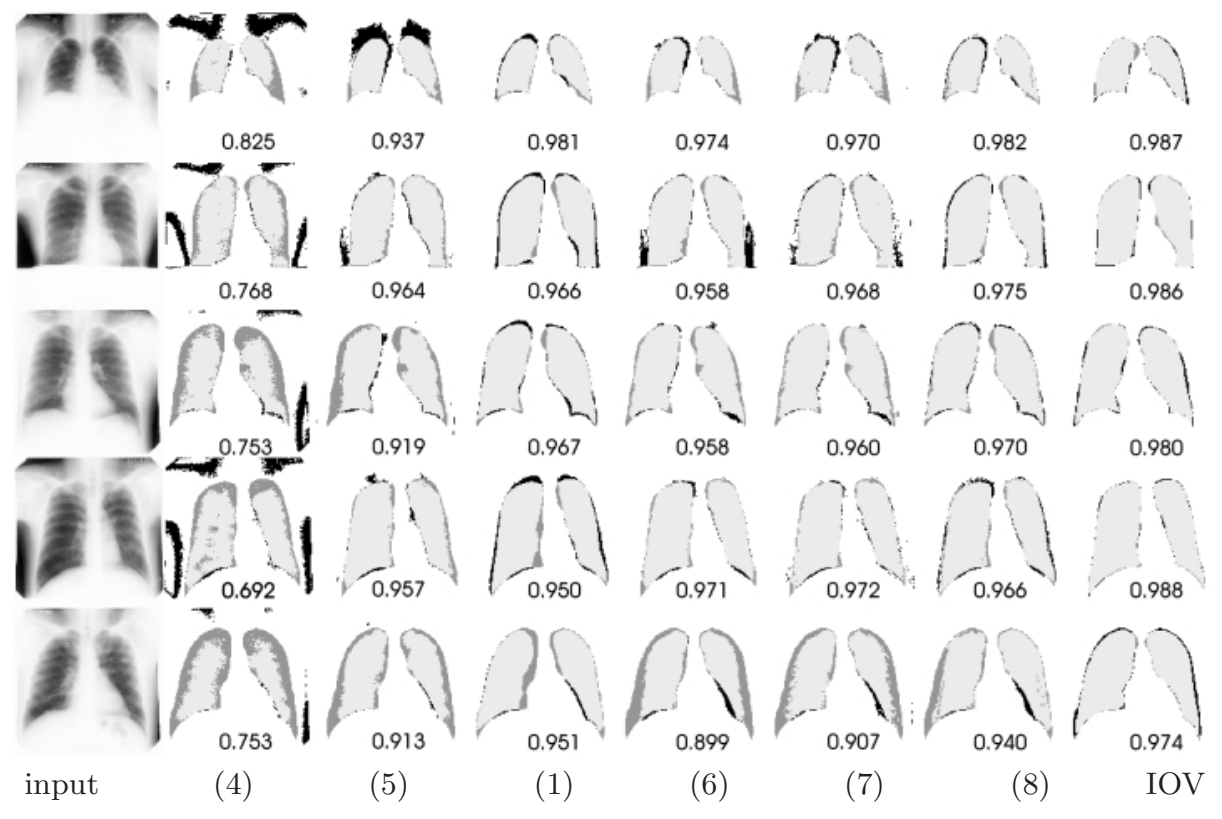

Fig. 2. Segmentation results for 5 radiographs of the test set for which system 8 gave respectively, best, good, average, poor and worst results. Systems are indicated in parentheses under each column. The right column shows the interobserver variability (a comparison of the segmentations of the first and second author). True negative pixels are shown white, true positive light gray, false positive pixels are dark gray and false negative pixels are shown in black.

differences in segmentation accuracy for normal versus abnormal radiographs. Key result is the accuracy of $96.9 \%$ of system 8 , which approaches the interobserver variability. Figure 2 shows results of several systems for 5 images. The images were chosen to range from best to worst performance for System 8, the overall most accurate system.

Using only intensity as feature, which can be seen as using an optimal multiple threshold, yields an accuracy $84.7 \%$. Vittitoe determined a single optimal threshold for his dataset, which scored $80.6 \%$. Using a neural network classifier with the intensities of a 3 by 3 neighbourhood (with the images subsampled to 64 by 64 pixels) of each pixel as input vector, he achieved $89.3 \%$ correct classification. The fact that our result with intensity as a single feature scores half-way between indicates that the performance increase of the 3 by 3 intensity classification versus fixed thresholding is partly due to the fact that a classifier determines multiple thresholds and partly due to the textural context information obtained from the pixel neighbourhood.

The interobserver variability, an accuracy of $98.4 \%$, provides a theoretical upper bound for the performance of any algorithm. This variability is mainly due to the difficulty in assessing the exact borders of mediastinum. Given the 


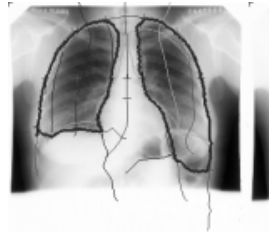

(a)

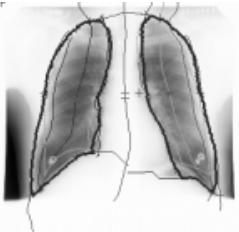

(b)

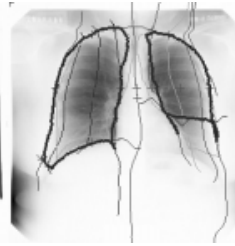

(c)

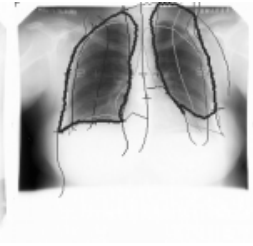

(d)

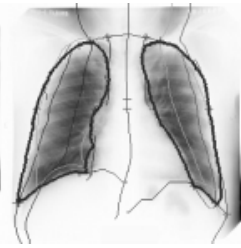

(e)

Fig. 3. Overview of occasional failures of the rule-based segmentation scheme. (a)-(b) Largely and slightly incorrect detection of left diaphragm due to the presence of stomach gasses (occurred in 18 of 115 cases). (c) Incorrect detection of left diaphragm due to a dense low left lung field. (d) Incorrect detection of the lung top. (e) Incorrect detection of the right mediastinum edge due to a pronounced right hilus.

performance of "simple" systems and the interobserver variability, a segmentation scheme for lung fields in chest radiographs will in practice have an accuracy between $90 \%$ and $98 \%$.

It is surprising that a classifier using intensity and the location of pixels as feature (system 5) already gives an accuracy of $93.3 \%$, especially if this is compared with the results of McNitt-Gray [7] who obtained 93.2\% using 59 features and spatial information. It might be partly explained by the difference in database. However, it may also indicate that, apart from spatial information, the use of many more local features than (raw) intensity, can hardly improve the discriminating power of pixel classifiers in segmenting lung fields.

The rule-based system is accurate and robust, but there is still room for improvements. We distinguish structural and occasional errors. The detected edge of left lung and mediastinum in the upper lung part is structurally too much to the right. Another example is the outer rib cage, which is detected as a line structure and located on the center of the overlapping ribs. The lung fields end slightly more medial. Because of the coarse scales used to detect the lung edges, their location has shifted. Figure 3 gives an overview of occasional errors. The most important is failure to correctly detect the left diaphragm. Indications for failure are differences between the average height of right and left diaphragm and the presence of diaphragm-like structures above/below the detected diaphragm (in case of unexpectedly low/high left diaphragms). Adding rules is the obvious way to extend a rule-based system and this has prompted $\mathrm{Xu}$ [3] to develop tests to classify thoraxes into several categories based on stomach gasses appearance.

The remaining pixel classifiers use the corrected location as feature. This is not sufficient to outperform the rule-based method. Therefore we attempted to combine the strengths of both methods in a system that reclassified those pixels for which rule-based segmentation and pixel classification with corrected location and intensity gave different results. This (system 8) increased the accuracy from 0.961 to 0.969 . This improvement may seem small, but in fact it bridges $35 \%$ of the gap between the rule-based scheme (96.1\%) and the maximally achievable 
interobserver variability of $98.4 \%$, and therefore may be considered a significant amelioration. Even more important is the increase of robustness. Reclassification is a means to correct those cases where the rule-based systems makes an occasional gross failure. If we consider the $10 \%$ of images that scored worst with the rule-based scheme, the average accuracy is $93.7 \%$. For the corrected scheme this percentage is $95.2 \%$. Note that these are different images, so for the 10 worst cases for the rule-based method, the performance gain is even larger. The worst case of all 115 images using the rule-based system scored $90.1 \%$, for the corrected scheme the worst result was $94.0 \%$.

Segmentation requires about 10 seconds for the hybrid scheme using a standard $350 \mathrm{MHz}$ PC. Our implementation is not optimized for speed. We expect that an improved implementation can segment a radiograph, even at the resolution used in clinical practice, within a second.

\section{Acknowledgements}

The authors are indebted to the radiologists Wallid Haddad, Ad van Gils, Erik Beek, and Paul van Waes. This work is supported by the IOP Image Processing, funded by the Dutch Ministry of Economic Affairs.

\section{References}

1. R. E. Bunge and C. L. Herman. Use of diagnostic imaging procedures: a nationwide hospital study. Radiology, 163:569-573, 1987.

2. X.W. Xu and K. Doi. Image feature analysis for computer-aided diagnosis: Accurate determination of ribcage boundary in chest radiographs. Medical Physics, 22(5):617-626, 1995.

3. X.W. Xu and K. Doi. Image feature analysis for computer-aided diagnosis: Detection of right and left hemidiaphragm edges and delineation of lung field in chest radiographs. Medical Physics, 23(9):1613-1624, 1996.

4. J. Duryea and J.M. Boone. A fully automatic algorithm for the segmentation of lung fields on digital chest radiographic images. Medical Physics, 22(2):183-191, 1995.

5. F.M. Carrascal, J. Carreira, M. Souto, P.G. Tahoces, L. Gomez, and J.J. Vidal. Automatic calculation of total lung capacity from automatically traced lung boundaries in postero-anterior and lateral digital chest radiographs. Medical Physics, 25(7):1118-1131, 1998.

6. M.F. McNitt-Gray, J.W. Sayre, H.K. Huang, and M. Razavi. A pattern classification approach to segmentation of chest radiographs. Proc. SPIE, 1898:160-170, 1993.

7. M.F. McNitt-Gray, H.K. Huang, and J.W. Sayre. Feature selection in the pattern classification problem of digital chest radiograph segmentation. IEEE Transactions on Medical Imaging, 14(3):537-547, 1995.

8. A. Hasegawa, B. Lo. Shih-Chung, M. T. Freedman, and S. K. Mun. Convolution neural network based detection of lung structures. Proc. SPIE, 2167:654-662, 1994.

9. O. Tsujii, M. Freedman, and K.M. Seong. Automated segmentation of anatomic regions in chest radiographs using an adaptive-sized hybrid neural network. Medical Physics, 25(6):998-1007, 1998.

10. N.F. Vittitoe, R. Vargas-Voracek, and E.F. Jr. Carey. Identification of lung regions in chest radiographs using markov random fiel modeling. Medical Physics, 25(6):976-985, 1998. 